\title{
再论生物多样性与生态系统的稳定性
}

\author{
王国宏 \\ (中国科学院植物研究所植被数量生态学开放研究实验室, 北京 100093 )
}

摘要: 本文在简述生物多样性与生态系统稳定性研究动态的基础上,从生物多样性和稳定性的概念出发,指出忽视 多样性和稳定性的生物组织层次可能是造成观点纷争的根源之一。特定生物组织层次的稳定性可能更多地与该 层次的多样性特征相关。探讨多样性和稳定性的关系应从不同的生物组织层次上进行。扰动是生态系统多样性 与稳定性关系悖论中的重要因子,如果根据扰动的性质,把生态系统 (或其他组织层次)区分为受非正常外力干扰 和受环境因子时间异质性波动干扰 2 类系统, 稳定性的 4 个内涵可以理解为 :对于受非正常外力干扰的系统而言, 抵抗力和恢复力是稳定性适宜的测度指标; 对于受环境因子时间异质性波动干扰的系统而言, 利用持久性和变异 性衡量系统的稳定性则更具实际意义。结合对群落和种群层次多样性与稳定性相关机制的初步讨论, 本文认为: 在特定的前提下, 多样性可以导致稳定性。

关键词：多样性, 稳定性, 生态系统, 群落, 种群

中图分类号:Q146 文献标识码：A 文章编号：1005-0094(2002)01-0126-09

\section{Further thoughts on diversity and stability in ecosystems}

WANG Guo-Hong

Laboratory of Quantitative Vegetation Ecology , Institute of Botany, Chinese Academy of Sciences , Beijing 100093

\begin{abstract}
The idea that greater diversity leads to increased community stability has long been contentious as a result of lack of consensus over the meanings of both diversity and stability in empirical studies and unrealistic models of communities in theoretical studies. Diversity and stability can occur in each biological structure level in ecosystems. However, only species diversity and community stability were involved in most of previous studies, which might be another major reason that there is no consensus on the issue. This paper presents an exploration of the relationship between diversity and stability of community and population, attaching great importance to the biological structure level of ecosystems in which diversity and stability are examined. We conclude, firstly, that prior to investigation on the relationship between diversity and stability, the biological structural level of both diversity and stability should be clearly identified, and some terms with respect to both diversity and stability should be recognized, such as phenotypic diversity, population diversity, species diversity, community diversity as well as population stability , community stability, ecosystem stability, etc. Secondly, four major properties included under the concept of stability, i. e. , resistance, resilience, persistence and temporal variability, all contribute to stability and yet may have very different relationships with diversity under different disturbance regimes. We redefine these properties of stability in terms of the characteristics of disturbances, dividing them into two types. Resistance and resilience are measurements of stability when ecosystems are disturbed by abnormal disturbances, such as fire, drought, grazing, attack by insects or diseases , invasion of exotic species, etc. Persistence and temporal variability are measurements of stability under normal environmental fluctuations. Thirdly, the dynamics of stability at a given level of ecosystem may be strongly influenced by the patterns of diversity within this level. For example, community stability may be favored by population
\end{abstract}


diversity as well as phenotypic diversity, while population stability ultimately depends on phenotypic diversity. Finally, with the discussions of related issues on diversity and stability in both community and population, this paper holds that, under specific premises, diversity may give rise to stability. Additionally , an assembly interpretation model with respect to the long-term debate over the issue on diversity and stability is proposed. We analogise diversity and stability as two assemblies, and three basic corresponding relations may exist among the elements between this two assemblies. The first two are efficient correspondence and inefficient correspondence. The former implies that diversity would lead to stability, the latter implies that it would not. The third relation is unknown correspondence. In this case, the properties underlying these corresponding relations are still unknown due to our limited knowledge. This simple model is promising to reconcile the long-term dispute over the issue on diversity and stability.

Key words : diversity , stability , ecosystem , community , population

人类活动导致全球环境变化,加速了生境丧失 和物种绝灭的速率, 对生态系统构成严重胁迫 ( Rapport \& Whitford ,1999; Pimm \& Raven ,2000; Tilman $2000 ;$;Howlett \& Dhand ,2000)。物种的丧失 或增加 (如外来种的入侵) 对生态系统的功能和稳 定性会造成怎样的影响，需要我们做出客观的评估 和预测 ( McCann 2000)。生物多样性与生态系统稳 定性这一古老的论题再一次成为生态学界关注的焦 点( Sankaran \& McNaughton ,1999 ; Tilman 2000 ; Loreau 2000)。

MacArthur ( 1955 ) 根据食物网理论提出稳定性 随着能量通路的增加而提高的论点。与 MacArthur 相类似,Elton(1958) 提出了生态系统越简单就越不 稳定的观点。此外, 多样性 - 稳定性假说还有其他 的表述形式, 如物种丰富的群落较物种贫乏的群落 具较高的稳定性（黄建辉，1994）; 物种的特征各不 相同, 物种多样性高的生态系统包含了能够抵御特 定环境扰动的物种，从而补偿了扰动对系统所造成 的影响。因此,生物多样性可以提高系统对扰动的 抵抗力 (McNaughton ,1977;Pimm,1984; Tilman \& Downing, 1994) 或增加生态系统的可靠性 (reliability) (Naeem \& Li , 1997)。Gardner \& Ashby (1970) 认为, 系统中的联结越大, 系统就越不稳定, 而高的 多样性总是与系统的分室结构和互作强度的减弱相 关联。Briand (1983) 通过对 40 个食物网相关分析 表明，随着物种多样性的增加，种间的互作强度和联 结度降低，说明复杂的食物网可使种群保持稳定。 多样性导致稳定性假说在 1970 年以前被认为是生 态学的中心法则、核心准则 ( Pimm, 1984;McNaughton ,1988) 或公理 ( Hairston et al. ,1968)。近年来许 多生态系统结构与功能的理论探索和实验生态学研
究成果也支持多样性 - 稳定性假说 (Naeem et al. , 1994 ; Tilman \& Downing, 1994 ; de Grandpre \& Bergeron, 1997 ;张知涁等, 1998;Grime,1998)。Til$\operatorname{man}(2000)$ 总结了近年来多样性与稳定性实验与理 论研究成果, 指出较高的多样性可以增加植物群落 的生产力、生态系统营养的保持力和生态系统的稳 定性。

早在 1968 年,Hairston 等对 MacArthur 的理论 曾提出过含蓄的质疑。尽管 Hairston 等所做的原生 动物和细菌实验结果部分地支持多样性导致稳定性 假说, 但他们也明确地指出 稳定性决不能仅仅根据 系统中能量通路的数量去决定。多样性导致稳定性 假说没有考虑各种专化的食物网，包括最高营养层 有多个物种共存的情形, 因此 MacArthur 的模型也 许仅仅是未来更全面的群落动态模型中的一个重要 组成部分 (Hairston et al. 1968)。May (1972,1973) 利用控制论理论 (cybernetic theory) 的概念构造了一 个理论观点。他指出, 系统复杂性的增加将削弱系 统的稳定性。对于自然世界的一般系统 (general system) , 种群的稳定性并不一定与营养的复杂性和 动植物区系的多样性相关, Elton 的 6 条论点是经不 起推敲的( May, 1972,1973)。

McCann (2000) 就多样性与稳定性研究提出了 一个新的思路。他指出, 既然现实的种群都是变化 的, 那么复杂群落的持续力在一定程度上可能依赖 于种群流 (population fluxes, 主要指群落密度的波 动)。于是, 无论生物过程还是非生物过程所导致 的种群变异, 都可以使物种以不同的方式去应对环 境的变化，从而削弱了潜在破坏性的竞争排斥对群 落的影响。基于此,McCann(2000) 提出了稳定性的 一个新解释 :即种群密度离极端密度越远 (越接近 
平衡密度), 稳定性就越高。多样性可以导致稳定 性, 但形成这种关系的驱动力不是多样性本身, 而是 群落包含物种或功能群的能力。McCann (2000) 认 为, 早期多样性与稳定性关系的争论中忽视了一些 根本性的因素。

Tilman (1996) 通过 207 个草地样地不同多样 性水平上生物量变异的长期研究, 指出多样性可以 导致群落和系统过程的稳定性, 但不会导致种群水 平上的稳定性。于是他认为该研究结论使多样性 稳定性假说与 May 观点的长期争论得到了调和。 Tilman (1996) 的结论很有启发性,但是应该注意 到，该结论的前提是以物种多样性去探讨各个层次 的稳定性, 如果以其他层次的多样性去认识相应层 次的稳定性，则可能是另外一种结果。因此，Tilman (1996) 关于多样性 - 稳定性假说与 May 观点调和 的结论是在非常有限的条件下得出的。

尽管多样性与稳定性理论探索已长达半个世 纪, 其间新的实验生态学证据不断积累, 新的理论学 说不断涌现, 但多样性与稳定性之间的关系仍然是 一个悬而未决的论题 (Pimm , 1984 ;Paine , 1988 ; de Grandpre \& Bergeron，1997)。Loreau (2000) 指出， 在这场争论中，一个主要的收获就是让我们意识到， 由于稳定性概念是如此的宽泛和模糊, 以至于不可 能得出一个强有力的理论概括。然而, 阐明多样性 与稳定性的关系毕竟具有重大的科学意义，同时对 生态系统管理和保护可提供理论指导 (Ehrlich \& Wilson，1991）。本文将对多样性和稳定性概念进 行简要评议, 以群落和种群层次为对象, 就多样性与 稳定性的关系表述初步观点。

\section{1 多样性与稳定性概念评议}

\section{1 多样性}

生物多样性是自然多样性程度的一个总称, 可 分为三个级系分类，即基因、物种和生态系统 (世界 资源研究所等,1993)。生态系统的各个生物组织 层次主要包括生态系统、群落、种群、个体、器官及基 因等。由于同一生物组织层次的不同单元均占有特 定的生态位，生态位的时空异质性决定了各个生物 组织层次的多样性。如果从生态系统功能角度出 发,能量流动和物质循环途径和方式是复杂多样的， 生态系统过程及功能必然具有多样化的特点。从物 种形成和发生的角度出发,物种过程或种群过程必
然存在多样性的内涵。可见, 多样性是一个内涵十 分丰富的概念。生态系统的各个生物组织层次在生 态系统结构的建成和功能发挥中有着特定的作用， 生态系统功能的保持和结构的完整可能与构成生态 系统各个生物组织层次的多样性特征密切相关。特 别地, 特定层次的稳定性特征可能更多地受制于相 应层次的多样性特征。如群落的稳定性与种群功能 型的多样性密切相关 (McCann, 2000), 而种群稳定 性根本上讲依赖于个体的生活史特征( Laurence， $2000)$ 。Loreau (2000) 指出, 生态系统的特定功能往 往受制于表型多样性 (phenotypic diversity) 的影响。 表型多样性的载体是功能型 (functional type), 而功 能型是超越系统层次 (即可以不拘泥于特定生物组 织层次) 的物种或种群组合。显然, 在多样性与稳 定性关系的探讨中, 就多样性而言, 决不能拘泥于一 个特定的层次 (如物种) 或该层次中的一个层面 (如 物种的丰富度)，应全面考察生态系统各个生物组 织层次及同一层次不同层面的多样性对系统稳定性 的影响。Tilman (1996) 关于草地群落多样性和稳 定性关系的研究是以物种丰富度变化去讨论生态系 统、群落和种群层次的稳定性特征, 其多样性的内涵 仍属物种多样性范畴, 从而影响了其结论的普适性。 目前在生物多样性与生态系统稳定性关系的讨论 中, 众说纷纭, 理论探索与实验研究之间难以达成共 识, 主要症结被认为是在实验生态学研究中稳定性 测度指标的不统一性 (de Grandpre \& Bergeron， 1997 ) 和在理论研究中模型群落的不真实性和模型 的过分简化等 (周集中,马世骏, 1990)。我们认为, 生物组织层次是生物多样性结构的重要内涵, 忽视 生物多样性的生物组织层次可能也是造成观点纷争 的重要根源之一。

\section{2 稳定性}

稳定性是指系统受到外部扰动后保持和恢复其 初始状态的能力, 是一个基于热力学原理的概念 (Hurd et al. ，1971)。生态系统稳定性的概念一般 包括抵抗力 ( resistance) 、恢复力 (resilience) 、持久性 (persistence) 和变异性 (variability) 等 4 个方面的内 涵( Pimm，1984)。在近年来的文献中, 还出现了诸 如可靠性 ( reliability) ( Naeem ,1998)、预测性 ( predictability) (McGrady-Steed et al. ，1997) 等概念, 但 从其属性上看，二者亦分别属于持久性和变异型的 范畴 (Loreau 2000)。概括起来, 稳定性内涵目前主 
要有两种界定 :其一把抵抗力和恢复力定义为生态 系统对外界干扰的响应, 持久性和变异性是两个描 述性概念, 没有涉及生态系统应付外界干扰的能力 ( 周集中,马世骏, 1990) ; 其二把抵抗力、恢复力、持 久性和变异性均描述为扰动后系统的反应 (黄建 辉,1994)。自然界的生物群落都存在着程度不同 的外力干扰，对稳定性内涵的两种界定中，前者过于 理想化，后者似乎更具合理性，但却没有反映出不同 性质扰动下系统响应的内涵。因此，如果根据扰动 的性质和强度，把生态系统 (或其他组织层次) 区分 为受非正常外力干扰 (如受火烧、异常干旱、水灾、 病虫害以及人类活动等) 和时间尺度上受环境因子 正常波动干扰的 2 类系统可能更具实际意义。基于 此, 稳定性的 4 个内涵可以理解为: 对于受非正常外 力干扰的系统而言, 抵抗力和恢复性是测度其稳定 性的主要指标;对于受环境因子正常波动干扰的系 统而言, 持久性和变异性是衡量系统稳定性的指标。 那些能适应环境因子的自然波动，并能保持其自身 生存与繁衍的系统就是稳定的生态系统, 如顶极植 物群落等。

Hastings 认为, 空间尺度也是与稳定性问题相 关联的一个重要因素,如同一个系统在不同的空间 尺度上稳定性特征是不同的。因此，在一些系统中， 稳定性是可以探讨的; 而在许多系统中, 稳定性也许 是一个不适合研究的问题 (Hastings , 1968,1988)。 稳定性测度的某一个指标不可能对处于不同性质扰 动下的所有系统都是适用的，但是如果根据扰动的 性质采用相应的测度指标去衡量, 则任何系统均存 在着稳定性的问题。因此,Hastings 的论点仅具部 分的正确性。McCann 对稳定性的定义提出了一个 新的解释, 即种群密度离极端密度越远 (越接近平 衡密度) 稳定性就越高, 变异性就越低。在野外生 态学研究中, 利用变异性的测度可以反映群落的稳 定性特征 (McCann,2000)。McCann 的定义中突出 了功能多样性 (种群密度波动) 对群落稳定性的作 用，这是值得肯定的，但却淡化了扰动性质在刻画稳 定性内涵中的作用。此外，仅仅通过变异性的测度 亦不能全面反映系统对扰动的响应机制。

稳定性的外延包括局域稳定性、全局稳定性、相 对稳定性和结构稳定性等 (黄建辉,1994)。该界定 中没有反映出稳定性的生物组织层次。在多样性和 稳定性实验及理论研究中往往以特定生物群落为研
究对象。然而, 稳定性的一些本质特征往往出现在 较低的 (群落以下) 生物组织层次上 (Hastings, 1988)。Tilman (1996) 曾在生态系统、群落和种群 层次上描述了各自的稳定性特征。Loreau (2000) 认 为种群层次的稳定性特征可能不同于群落及生态 系统层次的稳定性。事实上, 扰动胁迫可能会波及 特定生态系统或群落中的各个生物组织层次, 分别 探讨各层次对扰动的响应机制以及层次之间的相互 关系, 对客观地反映生态系统稳定性本质可能更具 积极意义。因此, 在稳定性的外延中应反映生物组 织层次的内涵, 如生态系统的稳定性、群落稳定性和 种群稳定性等。

\section{2 多样性与稳定性相关机理初探}

多样性和稳定性的外延十分丰富, 本文仅讨论 群落的种群多样性和种群内的多样性, 即表型多样 性( 包括个体、器官及繁殖对策等方面的多样性特 征) 对群落稳定性和种群稳定性的影响。

\section{1 多样性与抵抗力}

抵抗力是测度稳定性的一个重要指标。抵抗力 主要有以下几种描述: 系统抵抗外力干扰的能力 ( Tilman \& Downing ,1994) ; 扰动后一个系统保持在 一个平衡点的能力 (Connell \& Sousa,1983 ; Westman ,1978)。特定资源生产力水平下群落种群多样 性的高低直接影响到群落对扰动的抵抗力。对因外 来种入侵而引起的扰动, 种群多样性程度高的群落 由于缺乏闲置生态位, 外来种群难以立足; 而对于种 群多样性低的群落, 其空缺的生态位很容易被外来 种群入侵和占据。其结果可能有三种: 其一是外来 种群与原有种群在结构和功能上达成和谐, 形成较 稳定的群落; 其二是外来种群排斥原有种群, 使原来 的群落崩溃; 其三是原有种群排斥外来种群。第三 种可能性只有在原有种群的扩张力较大的情形下才 会发生。对火烧的扰动, 种群多样性高的植物群落 可能因包含具厚木栓层和含水率较高的抗燃树种而 抗火力较强。根据这一原理, 在人工林的建设中, 常 有计划地配植抗燃防火树种, 以增强人工群落的安 全性。就群落对害虫的抵抗力而言, 复杂的群落很 少发生爆发性的病虫害 (周集中, 马世骏, 1990 )。 因为物种多样性高的群落可以降低植食性昆虫的种 群数量, 而大规模单一植物物种的栽培, 无疑会使群 落结构单纯化, 容易诱发特定害虫的猖獗 ( 赵志模, 
郭依泉,1990)。因此,在农业生产实践中，多种作 物混交种植及采取多样化的种植方式是防止害虫爆 发的重要措施( Mackenzie et al. ，1998)。对危害青 海云杉 ( Picea crassifolia) 的青海云杉叶锈病 (Chrysomyxa qilianensis) 和云杉球果小卷蛾 (Cydia strobilella) 等 9 种害虫危害规律的研究表明,青海云杉 纯林的受害程度均高于与山杨 (Populus davidiana) 和华木 (Betula sp.) 混交的青海云杉种群 (刘兴聪， 1992)。对于干旱 (Tilman \& Downing ,1994 ;Tilman ， 1996 ) 和砍伐 (de Grandpre \& Bergeron ,1997) 的扰 动, 多样性程度高的群落同样表现出了较大的抵抗 力。物种多样性可能仅仅是一个表面的测度指标， 一个在较高物种多样性支撑下的植物群落, 必然具 有较高程度的表型多样性 (如多样化的种群生活史 对策和对扰动适应方式等), 后者可能是保持群落 稳定的直接因素。

Laurence et al. (2000) 指出, 种群稳定性从根本 上讲依赖于个体的生活史特征。种群多样化的生活 史对策本质上就是表型多样性。表型多样性高的种 群，必然提供了一个丰富的表型特征变异库。在经 历环境波动的过程中，对扰动抗性较强的表型可能 被选择而成为优势功能型, 使得种群过程和结构得 到了保持 (Loreau,2000)。Sankaran \& McNaughton (1999) 指出, 由于物种丰富度和物种相对多度变化 的不协调性，物种丰富的系统对扰动的抵抗力也许 有时较物种贫乏的系统低。换句话讲，一个物种丰 富度相对较低, 但种群的表型多样性较高的群落可 能较物种丰富度相对较高, 但表型多样性贫乏的群 落具有较高的抵抗力。此外,表型多样性丰富的种 群，不仅表现为形态性状的多样化，而且还具有多样 化的生理生化功能以及较完善的生态适应性, 在外 界环境的干扰下更容易做出较多的选择和应变，从 而有效地避免了整个类群的灭绝(王印政,1994)。 可见，种群表型的多样性提高了种群对扰动的抵抗 力。

\section{2 多样性与恢复力}

恢复力指群落经历扰动后恢复到初始状态的能 力。扰动前群落物种多样性水平的高低直接影响群 落的恢复力（Tilman \& Downing,1994), 因为它直接 决定了扰动后群落中种群构件和种子库的丰富度。 扰动对群落结构和功能的影响主要是打破了群落原 来的平衡, 导致群落生态位格局的紊乱。扰动后群
落的恢复主要是通过群落的种群构件组合对生态位 的再分配来完成的。物种多样性丰富的群落中, 具 有不同生物学特性和生态学特性的种群对某一特定 扰动的反映、受扰动影响的程度以及扰动后的恢复 情况各不相同, 扰动后的群落可能留下足以占有现 有生态位的构件。如在火烧和砍伐后, 物种丰富的 样地对干扰会作出迅速的反应, 主要是通过增加盖 度以阻止外来种的入侵, 进而逐步恢复其物种组成 和群落生产力 (de Grandpre \& Bergeron ,1997)。Loreau (2000) 在对生物多样性与生态系统功能的机 理解释中提出了选择效应 (selective effect) , 亦即高 的生物多样性提供了宽泛的变量范围。在扰动后, 特定的极端变量特征可能得到选择, 而该选择对群 落的恢复至关重要。如植物有效的繁殖对策对群落 经历诸如火烧、放牧等扰动后的恢复极其重要 ( Lavorel ,1999)。

种群受到扰动后恢复力的大小, 很大程度上取 决于种群表型多样性，如个体多样性、器官的多态性 及种群繁殖对策的多样性等。种群表型多样性水平 越高, 种群的恢复力也越强。如具有兼性营养繁殖 的种群, 如果扰动使种群的有性繁殖系统受损, 种群 还可以通过营养繁殖保持种群的延续。独叶草 (Kingdonia uniflora) 是只有一片叶子的矮小草本， 每个瘦果仅有一粒种子, 种子如因动物采食或败育 不能萌发, 它还可以通过发达的根状茎进行营养繁 殖, 种群仍保持了旺盛的生机。濒危植物长潒毛莨 泽泻 (Ranalisma rostratum) 的种群生殖对策表现出 了多样化的特点, 既能有性生殖, 又能产生无性小株 进行无性繁殖。在自然条件下, 由于春季较长时间 的水淹和夏秋杂草的竞争, 导致其种群数量下降而 极度濒危; 把植物移栽于迁地保护地的理想生境下, 相当于扰动解除后, 种群迅速增长( 陈家宽, 陈中 义,1999)。中国特有植物银杉( Cathaya argyrophylla) 的濒危机制研究表明, 人类活动特别是对森林 的乱砍滥伐加速了银杉种群的减少;而遗传多样性 低、生殖障碍大、种子产量少以及更新不良等银杉本 身固有的生物学特性对种群的生存构成了严重的威 胁 (谢宗强, 陈伟烈,1999)。种子在形态特征、休眠 期及寿命的长短、生理特性等方面的多样化, 为种群 在扰动后的恢复提供了坚实的物质基础。如云南松 (Pinus yunnanensis) , 尽管种群遭受像火烧这样的干 扰, 因其种子形态的多样性, 种群还是能够保存下来 
(黄瑞复，1992）。假连尧（Duranta repens）、栒子 (Cotoneaster sp.) 等植物的种子萌发极不整齐,假连 尧种子萌发过程可持续 2 个月;栒子种子于直播后 第 $1 、 2 、 3$ 年陆续出苗。种子在发芽期上表现出多样 化的特点对应付不良环境和繁衍后代是有利的（顾 增辉等,1998)。芽的多样性对种群的恢复力也具 有重要意义。如昆虫采食活动顶芽后, 休眠芽会萌 发，从而完成植株的形态建成。我们在甘肃张掖祁 连山北坡青海云杉林和祁连圆柏 (Sabina przewal$s k i i)$ 林演替系列上种群恢复状况的调查中发现,采 伐迹地上青海云杉林种群的恢复力明显较祁连圆柏 林强 (在海拔 $3000 \mathrm{~m}$ 处随机调查的 2 个样地中, 青 海云杉皆伐迹地幼苗天然更新达 14 株 $/ 100 \mathrm{~m}^{2}$; 祁 连圆柏仅为 3 株 $/ 100 \mathrm{~m}^{2}$ )。青海云杉林和祁连圆柏 林在经历扰动后, 种子是种群自然恢复的主要构件。 青海云杉种子在散布方式上具多样化的特点。它既 可以靠重力落下, 又可以借助风力飘散, 从而保证采 伐迹地更新所需的种源量。祁连圆柏种子不具飞翔 能力, 种子传播范围小, 在无母树的迹地上 (如火烧 迹地) 种群很难恢复。在自然界, 为什么有些植物 如危害农作物生长的各种田间杂草等, 人们试图斩 草除根却除而不尽;而有些植物 (如濒危植物) 在人 为干扰甚至在自然状态下就灭绝? 究其原因, 除毁 灭性的外力造成物种的灭绝外，在正常人为或自然 力的扰动下，物种兴衰取决于种群内的表型多样性 水平。此外，从多样性和稳定性的生物组织层次看， 单优群落的稳定性属于种群层次稳定性的范畴, 其 稳定性维持的机理同上，这里不再赘述。

\section{3 多样性与持久性和变异性}

持久性和变异性是两个不涉及系统对付外界干 扰能力的稳定性指标。事实上, 自然界不受外界干 扰的系统是不存在的, 这里所指不受干扰的系统是 相对于那些受到人为或非正常自然力干扰的系统而 言。显然,持久性和变异性是测度群落在正常的环 境变化中所表现出的群落特征, 亦即群落适应环境 正常波动的能力大小。就群落水平而言, 种群多样 性高的群落必然较多地包含了具有不同生物学和生 态学特性的种群，如前所述，抵抗波动的能力也强; 对于种群而言, 种群适应环境正常波动能力的大小 与种群内的多样性水平有关, 从根本上取决于种群 遗传多样性的丰富程度。近年来一些著名的实验生 态学的研究成果 ( Tilman \& Downing, 1994 ;Tilman，
1996 ; Naeem \& Li ,1997 ; McGrady-Steed et al. , 1997 ）虽然没有很充分地说明多样性对系统总体 稳定性的正面作用, 但就多样性可提高系统抵抗力 和降低时间尺度上的变异性等方面则提供了强有力 的证据 (Loreau 2000)。

3 关于多样性与稳定性理论探索中的观点 纷争

以 May 为代表的生态学家, 以模型群落为依据 建立数学模型, 研究了群落复杂性与稳定性的关系, 得出与多样性 - 稳定性假说完全相反的结论: 即系 统复杂性的增加将不可避免地削弱系统的稳定性 ( Gardner \& Ashby ,1970 ; May ,1972,1973 ; Goodman 1975;Pimm,1979)。但无论 May 的数学逻辑 和理论推导多么严密, 他的理论最大的缺陷是没有 考虑真实生态系统的调节机制 (黄建辉,1994)。根 据数学模型进行稳定性分析的基本前提是模型能描 述生态系统的动态变化, 以前研究所用的任何数学 模型能否描述生态系统的动态变化还值得怀疑 (周 集中, 马世骏, 1990)。数学方法为生态学的研究提 供了强有力的工具, 极大地促进了生态学的发展。 在人类探索生态系统本质的过程中，数学分析只有 尽可能地建立在真实生态系统的基础之上才可能反 映出系统的本质特征。但是作为理论探索, 模型群 落的构造是必不可少的, 它提供了一种思路和解决 问题的途径, 所得出的结论在特定条件下才能成立。 因此, 我们没有必要把理论与实验研究结果对立起 来, 相反应该整合和集成二者的研究成果，从而得出 一个更普遍的科学结论。

物种几余学说是生物多样性与生态系统功能理 论探索中的一个重要学说。Lawton \& Brown (1993) ,Vitousek \& Hopper(1993) 提出: 生态系统中 有许多种是相似的, 如果系统中主要功能群存在, 生 态系统的功能与多样性无关。这样, 物种几余就成 了生物多样性与生态系统功能之间关系的重要假说 之一(Naeem ,1998)。Walker (1992) 提出冗余概念 是出于评价生物保护的优先权的考虑, 即保护的重 点及优先权应放在对生态系统功能有独特贡献的物 种。这个观点的一个推论就是冗余种的保护是不必 要的, 这样冗余概念就与生物多样性的保护相抵触。 然而这并非 Walker 几余概念的本意( Walker ,1995; Gitay et al. , 1996 ,Naeem,1998)。冗余概念被认为 
是理解生物多样性生态价值的一个重要概念 ( Lawton \& Brown 1993;Vitousek \& Hopper 1993; ;itay et al. , 1996)。该假说也是保护生物学中优先保护原 则的理论依据, 而且在生态学界有着广泛的影响 (Chapin et al. , 1992 ;West , 1993 ;Bowman , 1994 ; Gitay et al. , 1996)。Gitay et al. (1996) 指出,由于 物种冗余概念的不确定性 (uncertainties) 和不实际 性 (impracticality) 将其应用于保护生物学中是危险 的。按照 Walker 的方法论, 要对物种几余存在的前 提条件和物种几余本身进行确定, 必须拥有每一个 物种至少是种群层次以上的相关资料, 而我们目前 对地球上绝大部分地区到底有多少物种都说不清， 更无法预测物种丧失后的影响, 试图保护所有的物 种和尽可能多的未受干扰的群落实乃明智之举 ( Gitay et al. 1996)。Tilman \& Dowing (1994) 认为,生 物群落的多样性对稳定性是至关重要的, 不支持冗 余假说。此外,Naeem (1998) 及党承林(1998) 依据 工程学自动控制系统可靠性理论中关于系统的备用 元件 (redundant components) 越多, 可靠性越高的原 则，指出应把物种冗余看作是生态系统的一个至关 重要的特征( Naeem,1998) 以及几余是以备用方式 来提高系统的稳定性 (党承林,1998)。Naeem (1998) 进一步指出,生物多样性的衰减意味着生态 系统物种冗余的丧失, 物种几余是功能群中物种的 丰富度所赋予的。可见，工程学可靠性理论中系统 的备用元件就是生态系统中生物多样性的对应物。 显然, 无论从生物多样性保护的角度还是从工程学 可靠性理论的角度出发, 生物群落的多样性对生态 系统稳定性是至关重要的。因而, 越来越多的学者 认为, 生物多样性是生态系统经历破坏性环境波动 时维持系统功能的生物保险 (Walker ,1995; Gitay et al. ,1996 ; Naeem ,1998; Loreau 2000)。

Tilman (1996) 通过草地群落不同多样性水平 上生物量变异的长期研究得出结论, 认为使多样性 - 稳定性假说与 May 观点的争论得到了调和。尽 管 Tilman (1996) 的研究结论是在有限的条件下得 出的, 同时也没有体现多样性的生物组织层次, 但其 结论具有启发意义。Loreau (2000) 也指出,稳定性 的诸多内涵与多样性的关系可能各不相同, 关键取 决于所考虑的变量。可见, 多样性和稳定性的关系 可能更多地表现在二者的特定组分之间。在此, 我 们提出一个多样性与稳定性关系悖论的集合论解释
(图 1) 如果把多样性与稳定性比作 2 个集合, 2个 集合中元素之间存在如下对应关系:1) 效应型对应 (多样性可导致稳定性), 如图中对称性对应有: A$\mathrm{A}^{\prime}, \mathrm{B}-\mathrm{B}^{\prime}, \mathrm{C}-\mathrm{C}^{\prime}$ ，D-D'，B-C'；非对称性对应有 :C$\left(\mathrm{B}^{\prime}, \mathrm{D}^{\prime}\right),(\mathrm{B}, \mathrm{C})-\mathrm{B}^{\prime},(\mathrm{B}, \mathrm{C}, \mathrm{D})-\mathrm{C}^{\prime},(\mathrm{C}, \mathrm{D})-\mathrm{D}^{\prime}$ 等 ; 2 ) 无效型对应 (多样性不会导致稳定性), 如 $A^{-}-B^{\prime}$, D-E'等 3 ) 未知型对应 (二者的关系不明)，如 B-A'， $E-E^{\prime}, E-D^{\prime}, F-F^{\prime}$ 等。效应型对应和无效型对应可 以共存, 不相互排斥。多样性导致稳定性假说可以 理解为 2 个集合元素之间的效应型对应关系, 相反 的观点可以理解为元素之间的无效型对应。导致观 点纷争的根源可能是我们尚未全面地认识到 2 个集 合间元素对应关系的多样性, 即把效应型对应与无 效型对应对立起来。此外, 如果多样性和稳定性集 合中的特定元素之间的对应关系尚未弄清或集合元 素仍然是残缺的, 也将影响 2 个集合对应关系的全 面认识。该集合模型告诉我们, 阐明多样性与稳定 性之间的关系, 必须明确二者的元素属性, 也就是本 文强调的多样性和稳定性的生物组织层次及其层 面。

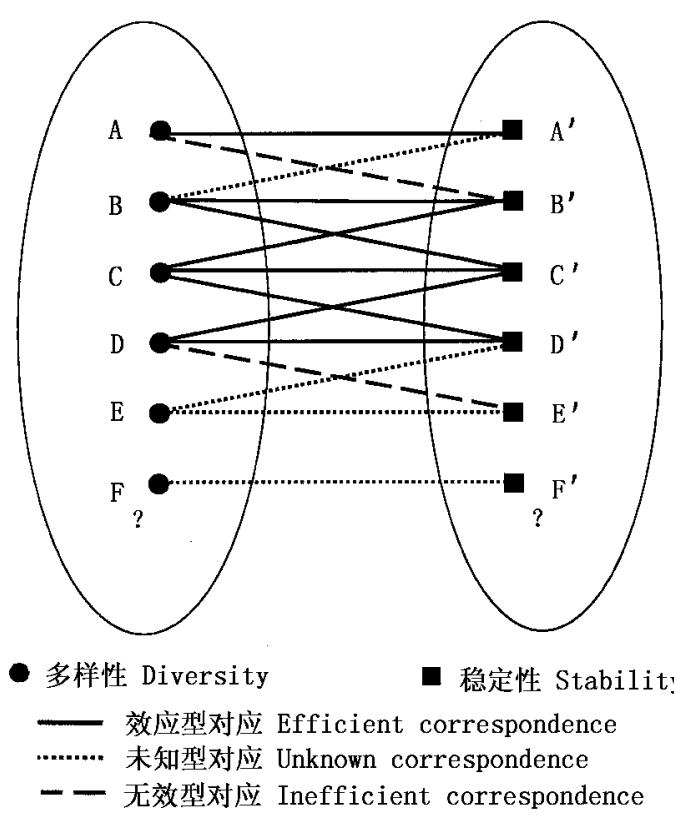

图 1 多样性与稳定性关系的集合论图解模型

Fig. 1 An assembly interpreting model with respect to the relationship between diversity and stability in ecosystems

\section{4 观点与结论}

通过群落和种群层次多样性与稳定性相关机制 的初步讨论, 本文认为多样性可以导致稳定性。该 
结论的成立有以下几个基本前提:1) 该格局存在于 特定的生物组织层次之间，2)多样性的内涵中不仅 包括了生态系统各生物组织层次的多样性特征, 而 且还包括表型多样性 (种内和种间) 3) 把抵抗力和 恢复力定义为受非正常外力干扰下的系统的稳定性 测度指标, 而变异性和持续性作为时间尺度上受环 境因子正常波动干扰下的系统稳定性测度指标;4) 就稳定性的生物组织层次而言, 本文讨论中所涉及 的层次是群落和种群, 群落的稳定性既受制于群落 种群的多样性, 又与种群内的多样性密切相关, 种群 稳定性则主要取决于种群的表型多样性;5)多样性 与稳定性讨论中所涉及的生物群落应该是充分发育 的成熟群落。根据本文提出的多样性与稳定性悖论 的集合论解释，我们认为充分理解和认识多样性和 稳定性 2 个集合中各元素属性及其相互关系,有望 成为调和多样性 - 稳定性关系悖论的突破口。

\section{参考文献}

陈家宽, 陈中义, 1999. 不同生境内濒危植物长喙毛莨泽泻 种群数量动态比较. 植物生态学报, 23(1):8 13

党承林, 1998. 植物群落的几余结构——对生态系统稳定性 的一种解释. 生态学报, 18(6): 665 672

顾增辉, 张金政, 冯桂强, 1998. 假连肴种子的休眠与萌发. 种子, 93( 1 ) : 49 50

黄建辉, 1994. 生态系统内的物种多样性对稳定性的影响. 见: 钱迎倩, 马克平 (主编), 生物多样性研究的原理与方 法. 北京: 中国科学技术出版社, $178 \sim 191$

黄瑞复, 1992. 云南松的种群遗传与进化. 云南大学学报 (自 然科学版), 14(2): 50 63

刘兴聪, 1992. 青海云杉. 兰州: 兰州大学出版社, 356 387 世界资源研究所, 联合国环境规划署, 联合国开发计划署, 1993. 世界资源报告. 北京: 中国环境出版社, 169

王印政, 1994. 绝灭及其与植物进化的关系. 见: 陈家宽, 杨 继 (主编), 植物进化生物学. 武汉: 武汉大学出版社, $328 \sim 364$

谢宗强, 陈伟烈, 1999. 中国特有植物银杉的濒危原因及保 护对策. 植物生态学报, 23(1): 1 7

张知彬, 王祖望, 李典谟, 1998. 生态复杂性研究——综述与 展望. 生态学报, 18(4) : 433 441

赵志模, 郭依泉, 1990. 群落生态学原理与方法. 重庆: 科学 技术出版社重庆分社, $170 \sim 172$

周集中, 马世骏, 1990. 生态系统的稳定性. 见: 马世骏 (主 编), 现代生态学透视. 北京: 科学出版社, 54 71

Briand F, 1983. Environmental control of foodweb structure. $E$ cology, 64: $253 \sim 263$

Chapin F S III, E D Schulze and H A Mooney, 1992. Biodiversity and ecosystem processes. Trends in Ecology and Evolution, 7: $107 \sim 108$

Connell J H W and P Sousa, 1983. On the evidence needed to judge ecological stability or persistence. American Naturalist, 121: $789 \sim 824$

de Grandpre L and Y Bergeron, 1997. Diversity and stability of understory communities following disturbance in the southern boreal forest. Journal of Ecology, 85: $777 \sim 784$

Ehrlich P R and E O Wilson, 1991. Biodiversity studies: science and policy. Science, 253: $758 \sim 762$

Elton C S, 1958. The Ecology of Invasions by Animals and Plant. Chapman and Hall, London, $143 \sim 153$

Gardner M R and W R Ashby, 1970. Connectance of large dynamic ( cybernetic) systems: critical values for stability. Nature, 228: 784

Gitay H, J B Wilson and W G Lee, 1996. Species redundancy: a redundant concept? Journal of Ecology, 84:121 124

Goodman D, 1975. The theory of diversity-stability relationships in ecology. The Quarterly Review of Biology, 50(3):237 266

Grime J P, 1998. Benefits of plant diversity to ecosytems: immediate, filter and founder effects. Journal of Ecology, 86: $902 \sim 910$

Hairston N G, J D Allan and R K Colwell, 1968. The relationship between species diversity and stability: an experimental approach with protozoa and bacteria. Ecology, 49: 1091 $\sim 1101$

Hastings A, 1968. The invasion question. Journal of Theoretical Biology, 121: $211 \sim 220$

Hastings A, 1988. Food web theory and stability. Ecology, 69: $1665 \sim 1668$

Howlett R and R Dhand, 2000. Nature insight biodiversity. $\mathrm{Na}$ ture, 405: 207

Hurd L E, M V Mellinger, L L Wolf and S J McNaughton, 1971. Stability and diversity at trophic levels in terrestrial successional ecosystems. Science, 173: $1134 \sim 1136$

Laurence D M, A Joshi and D J Borash, 2000. Does population stability evolve? Ecology, 81: $1273 \sim 1285$

Lavorel S, 1999. Ecological diversity and resilience of Mediterranean vegetation to disturbance. Diversity and Distributions, 5: $3 \sim 13$

Lawton J H and V K Brown, 1993. Redundancy in ecosystems. In: Schulze E D and H A Mooney (eds.), Biodiversity and Ecosystem Function. Springer-Verlage, New York, 255 270

Loreau M, 2000. Biodiversity and ecosystem functioning: recent theoretical advances. Oikos, 91:3 17

MacArthur R, 1955. Fluctuations of animal populations, and a measure of community stability. Ecology, 36:533 537

Mackenzie A, A S Ball and S R Virdee, 1998. Instant Notes in Ecology. BIOS Scientific Publishers Limited, UK, 255 260

May R M, 1972. Will a large complex system be stable? Nature, 238: $413 \sim 414$

May R M, 1973. Stability and Complexity in Model Systems. Princeton University Press, Princeton, 447

McCann K S, 2000. The diversity-stability debate. Nature, 405: $228 \sim 233$ 
McGrady-Steed J, P M Harris and P J Morin, 1997. Biodiversity regulates ecosystem predictability. Nature, 390: 162 165

McNaughton S J, 1977. Diversity and stability of ecological communities: a comment on the role of empiricism in ecology. American Naturalist, 111: $515 \sim 525$

McNaughton S J, 1988. Diversity and stability. Nature, 333: $204 \sim 205$

Naeem S, 1998. Speices redundancy and ecosystem reliability. Conservation Biology, 12:39 45

Naeem S, L J Thompson and S P Lawler, 1994. Declining biodiversity can alter the performance of ecosystems. $\mathrm{Na}$ ture, 368: $734 \sim 736$

Naeem S and S B Li, 1997. Biodiversity enhances ecosystem reliability. Nature, 390: $507 \sim 509$

Paine R T, 1988. Food webs: road maps of interactions or grist for theoretical development. Ecology, 69: $1648 \sim 1654$

Pimm S L, 1979. Complexity and stability: another look at MacArthur's original hypothesis. Oikos, 33:351 357

Pimm S L, 1984. The complexity and stability of ecosystem. Nature, 307: 321 326

Pimm S L and P Raven, 2000. Biodiversity: extinction by numbers. Nature, 403: $843 \sim 845$

Rapport D J and W G Whitford, 1999. How ecosystem respond to stress. BioScience, 49: $193 \sim 202$
Sankaran M and S J McNaughton, 1999. Determinants of biodiversity regulate compositional stability of communities. $\mathrm{Na}$ ture, 401: $691 \sim 693$

Tilman D, 1996. Biodiversity: population versus ecosystem stability. Ecology, 77(2):350 363

Tilman D, 2000. Causes, consequences and ethics of biodiversity. Nature, 405: $208 \sim 211$

Tilman D and J A Downing, 1994. Biodiversity and stability in grasslands. Nature, 367:363 365

Vitousek P M and D U Hopper, 1993. Biological diversity and terrestrial ecosystem biogeochemistry. In: Schulze E D and H A Mooney (eds.), Biodiversity and Ecosystem Function. Springer-Verlag, New York, $3 \sim 14$

Walker B H, 1992. Biodiversity and ecological redundancy. Conservation Biology, 6: $18 \sim 23$

Walker B H, 1995. Conserving biological diversity through ecosystem resilience. Conservation Biology, 9: $747 \sim 752$

West N E, 1993. Biodiversity of rangelands. Journal of Range Management, 46: $2 \sim 13$

Westman W E, 1978. Measuring the inertia and resilience of ecosystems. BioScience, 28: $705 \sim 710$

(责任审稿人 :张大勇; 责任编辑：时意专) 